\title{
Nitrogen retention mechanisms in tokamaks with beryllium and tungsten plasma-facing surfaces
}

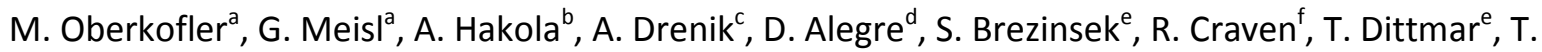

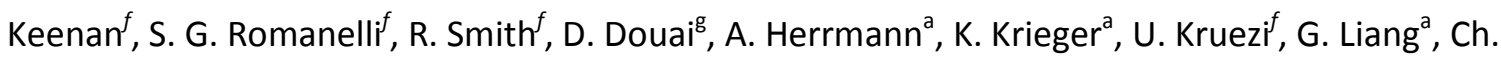 \\ Linsmeier ${ }^{a}$, M. Mozetic ${ }^{c}$, V. Rohde ${ }^{a}$, the ASDEX Upgrade team, the EUROfusion MST1 Team ${ }^{+}$and JET \\ Contributors* \\ EUROfusion Consortium, JET, Culham Science Centre, Abingdon, OX14 3DB, UK \\ ${ }^{a}$ Max Planck Institute for Plasma Physics, Boltzmannstraße 2, D-85748 Garching, Germany \\ ${ }^{b}$ VTT Technical Research Centre of Finland, Espoo, Finland \\ 'Jožef Stefan Institute, Jamova 39, 1000 Ljubljana, Slovenia \\ 'Laboratorio Nacional de Fusion, CIEMAT, Av. Complutense 40, 28040 Madrid, Spain \\ ${ }^{e}$ Forschungszentrum Jülich $\mathrm{GmbH}$, Institut für Energie- und Klimaforschung - Plasmaphysik, 52425 Jülich, \\ Germany \\ ${ }^{f}$ CCFE, Culham Science Centre, Abingdon, Oxon, OX14 3DB, UK \\ ${ }^{g}$ CEA Centre de Cadarache, 13108 Saint Paul lez Durance Cedex, France
}

\begin{abstract}
Global gas balance experiments at ASDEX Upgrade (AUG) and JET have shown that a considerable fraction of nitrogen injected for radiative cooling is not recovered as $\mathrm{N}_{2}$ upon regeneration of the liquid helium cryo pump. The most probable loss channels are ion implantation into plasma-facing materials, co-deposition and ammonia formation. These three mechanisms are investigated in laboratory and tokamak experiments and by numerical simulations. Laboratory experiments have shown that implantation of nitrogen ions into beryllium and tungsten leads to the formation of surface nitrides, which may decompose under thermal loads. On beryllium the presence of nitrogen has been seen to reduce the sputtering yield. On tungsten surfaces it has been observed that the presence of nitrogen can increase hydrogen retention. The global nitrogen retention in AUG by implantation into the tungsten surfaces saturates. At JET the steady state nitrogen retention is increased by co-deposition with beryllium. The tokamak experiments are interpreted in detail by simulations of the global migration with WallDYN. Mass spectrometry of the exhaust gas of AUG and JET has revealed the conversion of nitrogen to ammonia at percent-levels. Conclusions are drawn on the potential implications of nitrogen seeding on the operation of a reactor in a deuterium-tritium mix.
\end{abstract}

+ See http://www.euro-fusionscipub.org/mst1

* See the Appendix of F. Romanelli et al., Proceedings of the 25th IAEA Fusion Energy Conference 2014, Saint Petersburg, Russia 


\section{Introduction}

Impurity seeding is used in tokamaks with metallic plasma-facing materials with the aim to increase the radiated power fraction and mitigate otherwise excessive heat loads to the divertor targets [1]. Nitrogen is currently the preferred seeding species at JET and AUG because of its favorable radiative characteristics [2], the benevolent control behavior [3] and the observed beneficial effect on energy confinement $[4,5]$. However, nitrogen radicals are chemically reactive and can influence properties of the plasma facing surfaces and because of that affect the hydrogen (tritium) inventory build-up in the reactor vessel. It is therefore important to assess and understand the relative importance of the various nitrogen retention mechanisms in $\mathrm{N}_{2}$-seeded plasma discharges. In this contribution we review a selection of relevant laboratory and tokamak experiments and point out connected potential consequences and concerns for reactor operation in a deuterium-tritium mix.

\section{Surface nitrides on tungsten and beryllium}

Upon injection into the plasma nitrogen molecules are dissociated, ionized, transported along field lines and implanted into the plasma-facing surfaces. Fig. 1 shows the nitrogen depth profile after implantation into tungsten at room temperature with an impact energy of $2.5 \mathrm{keV}$ per atom [6]. The fluence of $2 \times 10^{16}$ $\mathrm{Ar} / \mathrm{cm}^{2}$ roughly corresponds to a depth of $10 \mathrm{~nm}$. Within this depth nitrogen is accumulated up to atomic concentrations of the order of 0.5 . Such high nitrogen concentrations are far beyond the solubility of nitrogen in tungsten [7] and are attributed to the formation of chemical bonds, i.e. of (amorphous) tungsten nitrides. Known stable stoichiometries are $\mathrm{WN}, \mathrm{WN}_{2}$ and $\mathrm{W}_{2} \mathrm{~N}$. The nitrides are formed by ion beam mixing, i.e. the energetic nitrogen atoms deliver the necessary energy to introduce defects in the metallic lattice and overcome the activation barrier for compound formation. The low diffusivity of the implanted nitrogen restricts the build-up of nitrogen concentrations beyond the solubility limit to a thin surface layer on the order of the penetration range of the energetic nitrogen ions in tungsten and the depth of the related damage profile. Investigations of the thermal stability of the nitrided surfaces have revealed a complex temperature behavior below a sample temperature of $900 \mathrm{~K}$ : The retained amount decreases steadily with increasing implantation temperature above $600 \mathrm{~K}$ [8]. Above $900 \mathrm{~K}$ decomposition of the compound is generally observed $[6,8,9]$.

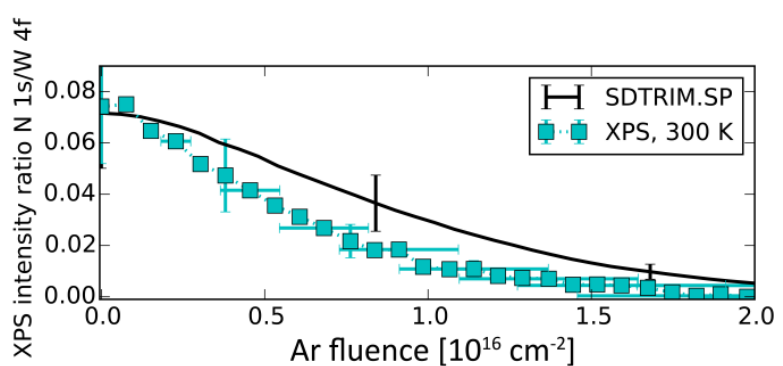

Fig. 1: Depth profile of nitrogen in tungsten analyzed by sputter X-ray photoelectron spectroscopy [6]. The tungsten surface had been bombarded at room temperature by mono-energetic $\mathrm{N}^{2+}$ ions at 5 keV to a fluence of $5 \times 10^{21} \mathrm{~N} / \mathrm{m}^{2}$. Ar ${ }^{+}$ions at $5 \mathrm{keV}$ were used for sputtering. The ratio of the $\mathrm{N} 1 \mathrm{~s}$ signal to the $\mathrm{W} 4 \mathrm{f}$ signal is plotted against the argon fluence. The experiment is compared to simulations using SDTrimSP combined with a forward calculation of the XPS signal intensities. In these simulations the 
atomic concentration of nitrogen was ad hoc limited to a maximum of 0.5 , corresponding to the stoichiometry of the tungsten nitride WN.

Analogously to the observations made on tungsten surfaces, also the implantation of nitrogen ions onto beryllium surfaces at room temperature leads to the formation of (amorphous) surface nitrides [10]. The saturation level of the nitrogen surface concentration as well as the observed shifts in the binding energies of the Be1s and N1s electrons (as observed by X-ray photoelectron spectroscopy) are consistent with the formation of stoichiometric beryllium nitride $\mathrm{Be}_{3} \mathrm{~N}_{2}$, which is a stable compound up to a temperatures beyond the melting point of Be (which lies at $1560 \mathrm{~K}$ ). No reduction of the nitrogen content in the implanted beryllium surface has been observed upon annealing up to $1000 \mathrm{~K}[10]$.

Due to the very limited depth of the nitrogen-enriched zone both on tungsten and on beryllium surfaces the nitrogen retention by ion implantation saturates with increasing fluence. The saturation fluence depends on the nitrogen to deuterium ratio in the incoming flux and on the ion energies. On tungsten at relevant nitrogen fractions and energies it is at most on the order of $10^{23}(\mathrm{D}+\mathrm{N}) / \mathrm{m}^{2}[6]$. The fluxes expected in ITER are on the order of $10^{20} \mathrm{D} / \mathrm{m}^{2} / \mathrm{s}$ at the main wall and up to $10^{24} \mathrm{D} / \mathrm{m}^{2} / \mathrm{s}$ in the divertor region close to the strike points [11]. Consequently the retention due to ion implantation into tungsten is likely to saturate very quickly in the divertor region. In long pulse operation saturation can be assumed to occur on all plasma-exposed surfaces.

Another consequence of the limited nitride thickness is that possible modifications of the properties of bulk material (such as melting point or electric and thermal conductivity) are negligible. The nitrogen accumulation at the surface can however strongly affect the interaction with the deuterium ions from the plasma. It has for example been shown that erosion rates of beryllium can be reduced $[12,13]$ and $G$. Meisl, this conference]. In the following another important example of a strong influence of a nitride surface layer is described.

\section{Increased deuterium retention in tungsten}

Upon exposure of tungsten surfaces to hydrogen plasmas or ion beams the hydrogen isotopes convey enough energy to overcome any surface barrier and thus penetrate the material. The fraction of implanted atoms that is retained in the material (both dynamically during the implantation as solute atoms in super-saturation or permanently due to trapping at defects) crucially depends on the diffusivity of hydrogen towards the bulk and towards the surface. It has been observed that the diffusivity of deuterium in tungsten nitride (at room temperature) is extremely low [14]. A reduced diffusivity (and/or solubility) of hydrogen in a thin surface layer can constitute a barrier for out-diffusing hydrogen when implantation occurs beyond this layer. Thereby the desorption flux can be reduced and the retention and/or the permeation to the rear surface of the sample (or to any interface at greater depth) can be increased.

Such an effect has been observed on tungsten samples that have been exposed sequentially to nitrogen and deuterium plasmas at $500 \mathrm{~K}$ [15]. Fig. 2 shows the topography of a tungsten surface after this sequential exposure (right image) compared to the exposure to only a deuterium plasma (left image). The appearance of blisters upon deuterium exposure is indicative of increased compressive stresses 
during exposure. This is attributed to a reduced out-diffusion of deuterium due to the surface nitride layer leading to an increased dynamic inventory during the exposure. In this experiment the nitrogen pre-implantation lead to increased deuterium retention by a factor of four. This is mainly attributed to trapping at defect sites in the surroundings of the blisters at depths between 0.5 and $5 \mu \mathrm{m}$ (i.e. far beyond the thickness of the surface nitride layer).
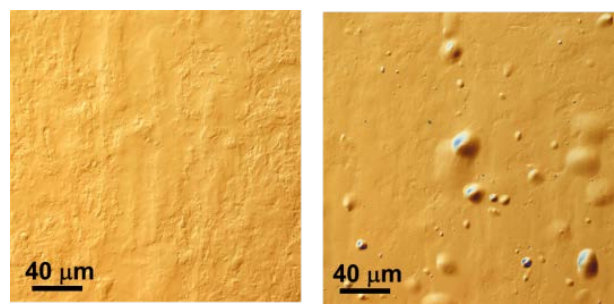

Fig. 2: Images acquired by optical differential interference contrast microscopy. Both surfaces have been exposed to deuterium plasmas at $500 \mathrm{~K}$ applying a bias of $-200 \mathrm{~V}$ and accumulating a fluence of $1.5 \times 10^{24}$ $\mathrm{D} / \mathrm{m}^{2}$. The right surface had been pre-exposed to nitrogen plasma at the same temperature and bias, accumulating a fluence of $1.5 \times 10^{22} \mathrm{~N} / \mathrm{m}^{2}$ [15].

[15]The tungsten surfaces in ITER will feature a broad distribution of temperatures and it has been predicted that (without the presence of nitrogen) the largest part of the hydrogen inventory in a tungsten divertor will build up in areas with intermediate fluxes and temperatures around $500 \mathrm{~K}$ [16]. The presence of surface nitrides would further increase the contribution of these areas to hydrogen retention. Further laboratory investigations of this effect upon simultaneous exposure to hydrogen and nitrogen ions (at various relative energies, i.e. various relative implantation ranges) or mixed plasmas would be of interest.

\section{Co-deposition of nitrogen with beryllium}

The term co-deposition stands for the formation of mixed material layers by implantation of a volatile species into a continuously growing deposit of a solid material. This mechanism strongly contributes to fuel retention in all fusion devices with low-Z plasma facing materials $(\mathrm{Be}, \mathrm{C})$ and is predicted to be the dominant mechanism governing the long term tritium retention in ITER. The findings of [17], [18] and [19] as well as the results on the effect of surface roughness presented in this paper show that nitrogen can be retained in a similar fashion. This is especially true when beryllium is present as a plasma-facing material because of its comparatively high erosion yield and the above-mentioned thermal stability of beryllium nitride. 


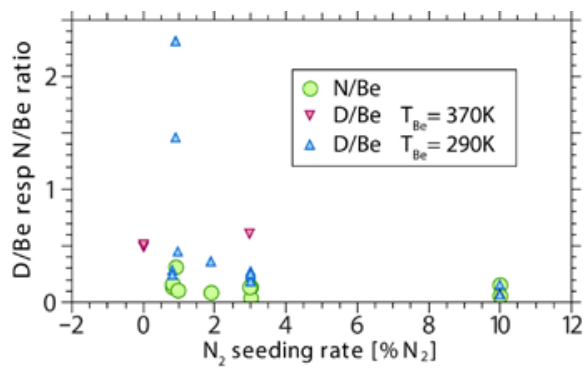

Fig. 3: Concentrations of nitrogen and deuterium in beryllium co-deposits collected in PISCES-B (measured by Rutherford backscattering spectrometry) as a function of the admixture of nitrogen to the deuterium plasma. The collection plate was kept at room temperature. Plasma parameters and bias voltages at the erosion target vary considerably between the data points [17].

Some insight into the nitrogen uptake in beryllium co-deposits has been gained by the exposure of beryllium samples to mixed deuterium-nitrogen plasmas in PISCES-B [17]. Co-deposited material was collected on witness plates and analyzed by ion beam analysis to quantify its elemental composition. Fig. 3 shows the nitrogen content in the co-deposits. The scatter in the data is large because the deposits have been produced in a range of different plasma parameters. However, the resulting nitrogen to beryllium ratios lie in the range of 0.05 to 0.35 and do not have a clear dependency on the nitrogen fraction in the gas admitted to the plasma. These ratios are in line with the analysis of co-deposits in JETILW $[18,19]$.

These nitrogen concentrations are comparable or larger than typical deuterium concentrations in beryllium co-deposits [20], [M. Mayer, this conference]. It can be concluded, that the uptake of nitrogen in co-deposits is an efficient mechanism for nitrogen retention. Since co-deposits can grow continuously without a saturation effect, it is expected that this retention mechanism will dominate over nitrogen implantation into plasma-facing materials in long discharges with nitrogen seeding.

\section{Nitrogen implantation and co-deposition in AUG}

In tokamaks the toroidal and poloidal distribution of the nitrogen content in the first wall is governed by migration, i.e. by the continuous ion implantation, re-erosion and re-implantation. The WallDYN code has been developed to model these processes in an integrated manner [21]. This code has been benchmarked against experiments at AUG [G. Meisl et al., submitted to Nucl. Fusion] and applied to estimate the time evolution of the global nitrogen retention in AUG [22]. The simulation (Fig. 4) shows that the retention of nitrogen is initially close to $100 \%$ of the injected amount due to implantation into pristine tungsten surfaces. However, the retention saturates already within a few seconds, which ultimately limits the nitrogen uptake into the vessel walls to roughly $5 \times 10^{21}$ atoms. 


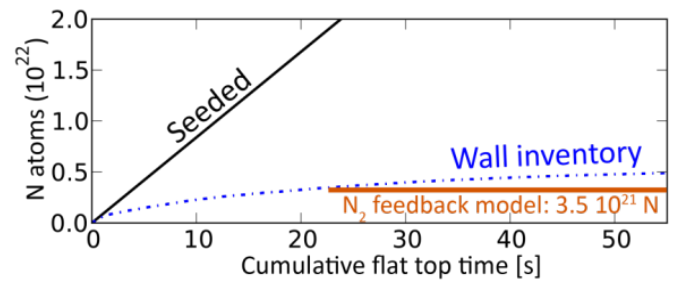

Fig. 4: WallDYN simulation of the nitrogen wall inventory build-up in a seeded discharge in AUG [22] and comparison to the value used for the nitrogen feedback control at AUG [3].

At AUG a phenomenological model has been used to determine the parameters of the feedback loop that is employed for the control of the nitrogen seeding rate [3]. This model assumes the total nitrogen inventory in AUG to saturate at $3.5 \times 10^{21}$ atoms, which is comparable to the value obtained in WallDYN simulations. However, there are still notable uncertainties: The two models make different assumptions on the plasma wetted area and a considerable influence of surface roughness on the saturation nitrogen areal density had to be assumed in the feedback model. In the following the influence of surface roughness on nitrogen retention is discussed.

\section{Influence of roughness}

To investigate the influence of surface roughness on the accumulated nitrogen areal density fine grain graphite samples of various roughnesses were prepared. Fig. 5 (left) shows a photograph of the three different types of samples that were employed: Polished samples with a roughness value $R_{a}$ around 0.03 $\mu \mathrm{m}$, samples without any treatment after the production by milling with $R_{a}$ of roughly $1 \mu \mathrm{m}$ and samples with surfaces deliberately roughened by sandblasting with hard spar yielding an $R_{a}$-value of a few $\mu m$. These samples were coated with 20 to $25 \mathrm{~nm}$ of tungsten by arc-discharge deposition, inserted into dedicated notches on two custom outer divertor target plates of AUG and exposed on the new divertor manipulator DIM-II of AUG [23] to 13 L-mode discharges with $\mathrm{N}_{2}$-injection and one without. Further details on the plasma scenario can be found in [A. Hakola, this conference].

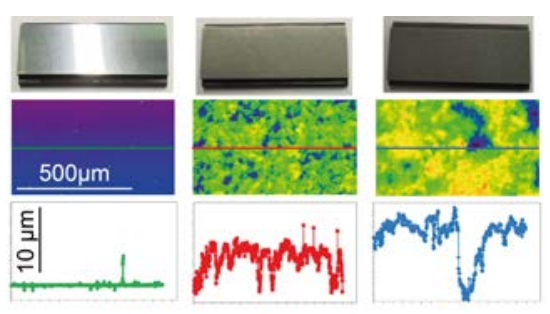

Fig. 5: Top: Photographs of the three types of samples used in the roughness investigation. From left to right: A polished, a milled and a sandblasted graphite sample prior to the coating with tungsten.

[23]

Middle and bottom: Confocal laser scanning microscopy measurements on the polished, milled and sandblasted surfaces. The profile scans shown in the bottom row correspond to the data acquired along the line indicated in the respective maps in the middle row. 
The exposed surfaces were analyzed for their elemental composition by nuclear reaction analysis. Fig. 6 shows the resulting poloidal nitrogen and boron distributions along the outer divertor target tiles.

(Differences in surface roughness can influence the depth resolution of such measurements. But they have no influence on the plotted depth-integrated areal densities.) The poloidal profiles on the milled and the sand-blasted surfaces feature clear peaks a few $\mathrm{cm}$ upstream of the nominal strike line position. On the polished sample the measured nitrogen and boron distributions are comparatively flat and smaller by a factor of 4 to 5 than the peak values in the case of the rough samples.

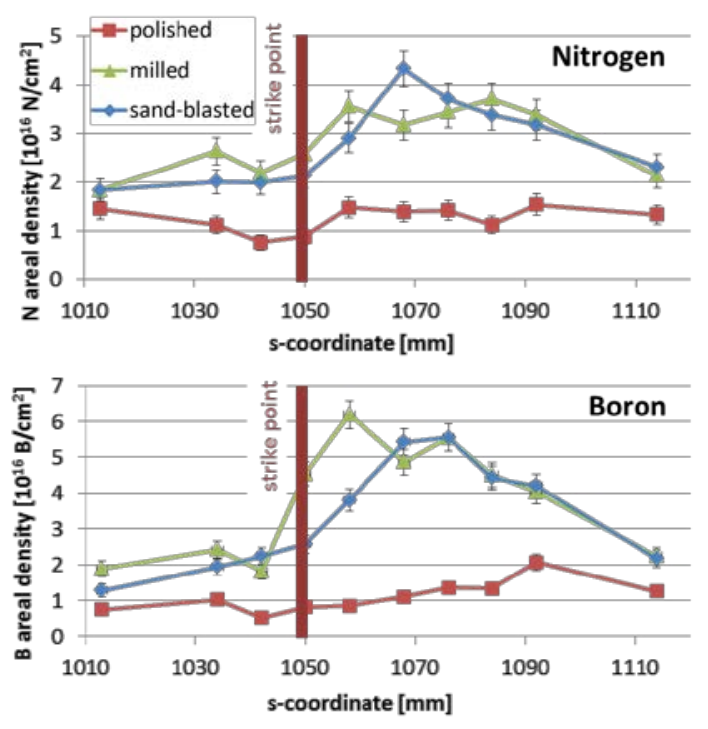

Fig. 6: Nitrogen (top) and boron (bottom) areal densities on the tungsten coated graphite samples after exposure to a series of N2-seeded discharges at the outer divertor of AUG. The nitrogen and boron content was determined from nuclear reaction analysis (NRA) and is plotted as a function of the poloidal $\mathrm{s}$-coordinate. The strike point position is indicated by the red bar. The error bars are calculated from the NRA counting statistics.

A number of plausible explanations exist for this considerable difference in nitrogen (and boron) deposition depending on surface roughness. For example, a change in roughness implies a change in the distribution of angles of incidence of the impinging ions. Also, retention of nitrogen in shadowed slopes can occur by implantation or co-deposition of re-eroded nitrogen with boron or carbon [24]. The temperature experienced by deposits might be reduced in deep valleys of the rough surfaces. This would lead to a reduced outgassing from co-deposits with increasing depth of the valleys, i.e. with increasing roughness. Further analysis of the exposed surfaces (e.g. by electron microscopy) will shed some more light on the effects of roughness.

\section{Nitrogen retention in JET and ITER}


A global gas balance experiment in a $\mathrm{N}_{2}$-seeded ohmic L-mode scenario (plasma current 2.0 MA, toroidal field 2.2 T) was performed at JET in 2011 by regenerating the divertor liquid $\mathrm{He}$ cryo panel before and after a series of equivalent discharges. Of the injected nitrogen atoms $52.5 \%$ were not recovered in this experiment and the observed deuterium retention was 3.7\%, which is higher by a factor of 2 to 3 compared to similar non-seeded scenarios. The amount of missing deuterium in the gas balance provides an upper limit for the fraction of nitrogen atoms that can have been converted to ammonia: Subtracting a retention of $2 \%$ as typically observed in similar non-seeded scenarios leads to an additional deuterium retention of $1.7 \%$ due to the presence of nitrogen. This corresponds to an upper limit for the conversion fraction of nitrogen atoms to ammonia molecules (averaged over the discharges of this experiment) of $15 \%$. Any increase in hydrogen retention compared to non-seeded discharges that can be attributed to increased diffusion to the bulk in tungsten (c.f. tungsten-nitride formation above) and/or to an increased amount of hydrogen stored in co-deposits would decrease the value of this upper limit.

\section{Implantation and co-deposition}

Post-mortem analyses of surfaces exposed in JET-ILW have confirmed considerable nitrogen fractions in co-deposits $[18,19]$ : The nitrogen-to-beryllium ratios ranged from 0.03 to 0.15 on the inner divertor tiles. On deposition probes in the outer divertor they were in the range of 0.04 and on first mirrors roughly 0.3. An extrapolation of these measurements to the whole vessel surface and an assessment of the fraction of injected nitrogen that is retained by co-deposition have not yet been attempted.

A WallDYN simulation has been performed for JET-ILW, employing a similar plasma scenario as that used for the above-mentioned gas balance experiment and puffing the same number of nitrogen atoms [G. Meisl, this conference]. The resulting nitrogen retention by implantation and co-deposition with beryllium amounts to $24 \%$ of the injected atoms. The formation of ammonia is currently not considered in these simulations. The calculation is subject to considerable uncertainties. Still, the result implies that only about half of the $52.2 \%$ of nitrogen missing in the mentioned gas balance experiment performed in 2011 can be explained by implantation and co-deposition and that ammonia formation could account for the other half.

\section{Ammonia formation}

The presence of (tritiated) ammonia in the exhaust gas of ITER can have implications on the duty cycle of the reactor (due to the necessity of more frequent cryo pump regenerations to higher temperatures) as well as on the design of the tritium plant. The concentration of ammonia in the exhaust gas of $\mathrm{N}_{2}$-seeded discharges in current tokamaks can be measured by mass spectrometry analysis. Such an analysis is not straightforward for plasma discharges in deuterium because of overlapping signals from deuterated water, methane and ammonia [25]. It is further complicated due to the tendency of ammonia to perform isotope exchange reactions with hydrogen (protium) atoms in the pump ducts surfaces [26, 27]. A code has been developed for decomposing mass spectra from partly deuterated molecular species. However, due to uncertainties in the cracking patterns the solutions found by this code are not always unique. To overcome this difficulty, dedicated experiments have been performed in the hydrogen (protium) campaign C34 at JET-ILW. This simplifies the analysis of mass spectra (as well as the numerical decomposition) because the dominant signals from the fully protonated molecules $\mathrm{H}_{2} \mathrm{O}, \mathrm{CH}_{4}$ and $\mathrm{NH}_{3}$ 
appear at different mass-to-charge ratios (namely 18, 16 and $17 \mathrm{amu} / \mathrm{e}$, respectively). The decomposition code was applied to the data from this experiment in protium. It consistently revealed fully protonated species of water, methane and ammonia as dominant fractions with values for the HD ratio $\mathrm{H} /(\mathrm{H}+\mathrm{D})$ between 0.95 and 1.0.

For simplicity the HD-values have been fixed at 1.0 in the decomposition analysis displayed in Fig. 7. The figure shows the resulting fractions of nitrogen atoms converted to ammonia molecules in a series of equivalent discharges (Ohmic L-mode at 1.8 MA plasma current and 1.8 T toroidal field). All discharges were $\mathrm{N}_{2}$-seeded except for the first and the third. It can be seen that the ammonia level increases from discharge to discharge reflecting the legacy of nitrogen (adsorbed, as nitride or as ammonia) in the vessel that has also previously been observed at JET $[28,29]$. Although a legacy from previous discharges is clearly present, some wall depletion and outgassing appears to occur in the ramp-down and ramp-up phases as well as in-between discharges. As a consequence the ammonia levels in the beginning of a seeded discharge are lower than at the end of the flat-top of the preceding equivalent discharge. No saturation of the ammonia conversion rate is observed within a single $\mathrm{N}_{2}$-seeded discharge. The highest conversion fractions observed in the flat-top phase of the discharges reach $0.4 \%$. These results are in line with experiments at AUG [25], which also showed that post-discharge outgassing strongly contributes to the amount of ammonia produced in a single discharge.

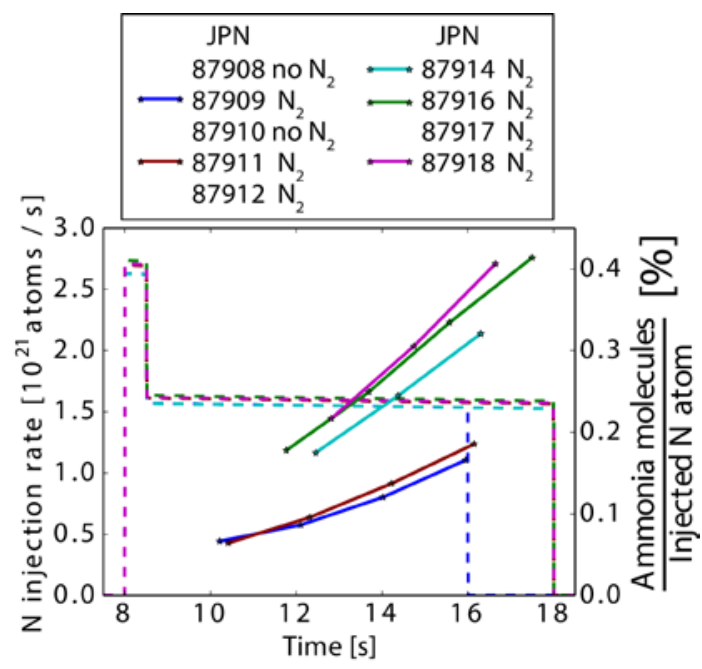

Fig. 7: Nitrogen injection rate and fraction of ammonia molecules per injected nitrogen atom in a series of equivalent JET discharges. All successful discharges of the session are included in the legend, but only the ones with useful mass spectrometry data appear in the graph. The analysis is restricted to a time frame with negligible variations in plasma density and sub-divertor pressure in order to exclude artifacts caused by partial variations on the time-scale of the acquisition of one mass spectrum, which is about $1.9 \mathrm{~s}$.

Before and after this experiment both the liquid helium and the liquid nitrogen $\left(\mathrm{LN}_{2}\right)$ cryo panel of the JET divertor cryo pump were regenerated. Fig. 8 shows the result of the decomposition of the mass spectra recorded upon increasing the temperature of the liquid nitrogen panel after the experiment. Again fully protonated molecules are the predominant fractions in the exhaust gas (as expected). Quantification of the amounts released from the cryo panel is hampered by relatively high pressures 
being reached in the ducts behind the turbo molecular pumps: A backflow of gas cannot be excluded. At the time point of the observed maximum in the ammonia and methane partial pressures the $\mathrm{LN}_{2}$ feed was opened again causing parts of the cryo panel to start cooling down and thus increasing the pumping speed. The evolution of the pumping speed after the maximum is therefore also not known and the pumped amount cannot be calculated from this partial pressure time trace.

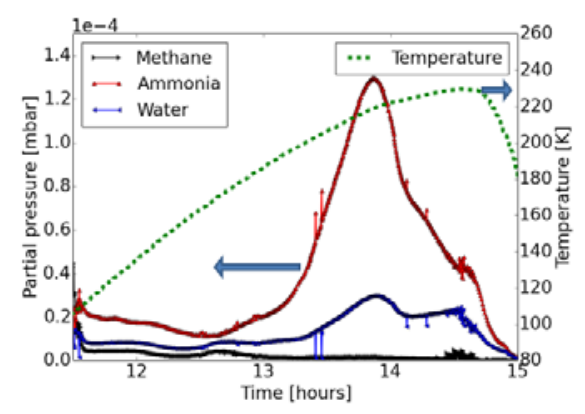

Fig. 8: Time traces of partial pressures and temperature upon regeneration of the $\mathrm{LN}_{2}$ cryo panel after a series of $\mathrm{N}_{2}$-seeded discharges. The partial pressure values were derived by decomposition of the acquired mass spectra with their sum absolutely calibrated against the sub-divertor baratron pressure. The plotted temperature represents a local measurement on one of the chevrons which shield the the $\mathrm{LN}_{2}$ lines.

Still, one can calculate a lower bound of the ammonia released from the $\mathrm{LN}_{2}$ panel from the maximum partial pressure reached during the regeneration: Multiplication of the peak pressure with the volume of the JET vessel $\left(183 \mathrm{~m}^{3}\right)$ yields 25 mbar*liters of ammonia. This corresponds to at least $0.44 \%$ of the nitrogen atoms injected into the plasma being converted to ammonia molecules.

\section{Summary and conclusions}

The main mechanisms for nitrogen retention in $\mathrm{N}_{2}$-seeded tokamak discharges could be identified to implantation into plasma-facing materials, co-deposition (with $\mathrm{Be}, \mathrm{B}$ or $\mathrm{C}$ ) and the formation of ammonia. Implantation is dominant in the early stage of a discharge. However, this mechanism saturates at elevated fluences due to the limitation of the nitrogen accumulation to a very thin surface layer on the order of the ion range. Laboratory experiments at beryllium surfaces have shown that the formation of this thin nitride layer can reduce the erosion rate. On tungsten the nitride layer acts as a permeation barrier which (in the case of sequential nitrogen and deuterium implantation at $500 \mathrm{~K}$ ) has been shown to potentially increase deuterium retention and blistering.

Recent results show that in JET-ILW and ITER on long time scales co-deposition with beryllium dominates over implantation. At AUG a considerable influence of surface roughness on the nitrogen areal density accumulated on tungsten surfaces has been observed, which is attributed to co-deposition with boron or carbon in valleys and pits. 
A conversion factor of nitrogen atoms to ammonia molecules on the order of $5 \%$ (including outgassing) has previously been reported for AUG [25]. At JET steady state was not yet achieved in the current investigations. At JET the conversion factor was estimated from measurements in the flat top phase of discharges as well as from the regeneration of the liquid nitrogen cryo panel after a series of $\mathrm{N}_{2}$-seeded discharges. The resulting values in the range of $0.4 \%$ have to be considered as lower bounds. The actual conversion could be in the same range as observed at AUG. Most of the ammonia can be assumed to be pumped by the cryo pumps. However, due to the strong sticking behavior of ammonia an inventory of (tritiated) ammonia can build up on all inner surfaces and in the pump ducts. These findings may imply the necessity of a design upgrade of the ITER tritium plant to make it compatible with the expected amounts of ammonia in the exhaust gas. The presence of ammonia might also influence the required regeneration rate of the ITER cryosorption pumps. Finally, it was shown that ammonia influences the outgassing and pump-down behavior. A possible impact on the duty cycle of a future power plant should be assessed.

\section{Acknowledgement}

This work has been carried out within the framework of the EUROfusion Consortium and has received funding from the Euratom research and training programme 2014-2018 under grant agreement No 633053. The views and opinions expressed herein do not necessarily reflect those of the European Commission.

\section{References}

[1] A. Kallenbach, R. Dux, M. Mayer, R. Neu, T. Pütterich, V. Bobkov, J.C. Fuchs, T. Eich, L. Giannone, O. Gruber, A. Herrmann, L.D. Horton, C.F. Maggi, H. Meister, H.W. Müller, V. Rohde, A. Sips, A. Stäbler, J. Stober, and ASDEX Upgrade Team. Non-boronized compared with boronized operation of ASDEX Upgrade with full-tungsten plasma facing components. Nucl. Fusion, 49 (4): 045007, 2009. URL http://stacks.iop.org/0029-5515/49/i=4/a=045007.

[2] A. Kallenbach, M. Balden, R. Dux, T. Eich, C. Giroud, A. Huber, G.P. Maddison, M. Mayer, K. McCormick, R. Neu, T.W. Petrie, T. Pütterich, J. Rapp, M.L. Reinke, K. Schmid, J. Schweinzer, and S. Wolfe. Plasma surface interactions in impurity seeded plasmas. J. Nucl. Mater., 415 (1, Supplement), August 2011. ISSN 0022-3115. URL http://www.sciencedirect.com/science/article/pii/$\underline{\mathrm{S} 0022311510010652 .}$.

[3] A. Kallenbach, R. Dux, J.C. Fuchs, R. Fischer, B. Geiger, L. Giannone, A. Herrmann, T. Lunt, V. Mertens, R. McDermott, R. Neu, T Pütterich, S. Rathgeber, V. Rohde, K. Schmid, J. Schweinzer, W. Treutterer, and ASDEX Upgrade Team. Divertor power load feedback with nitrogen seeding in ASDEX 
Upgrade. Plasma Phys. Control. Fus., 52 (5): 055002, 2010. URL http://stacks.iop.org/0741-3335/52/i=5/$\underline{\mathrm{a}=055002}$.

[4] J. Schweinzer, A.C.C. Sips, G. Tardini, P.A. Schneider, R. Fischer, J.C. Fuchs, O. Gruber, J. Hobirk, A. Kallenbach, R.M. McDermott, R. Neu, T. Pütterich, S.K. Rathgeber, J. Stober, J. Vicente, and the ASDEX Upgrade Team. Confinement of 'improved H-modes' in the all-tungsten ASDEX Upgrade with nitrogen seeding. Nucl. Fu, 51 (11): 113003, 2011. URL http://stacks.iop.org/0029-5515/51/i=11/a=113003.

[5] G.P. Maddison, C. Giroud, B. Alper, G. Arnoux, I. Balboa, M.N.A. Beurskens, A. Boboc, S. Brezinsek, M. Brix, M. Clever, R. Coelho, J.W. Coenen, I. Coffey, P.C. da Silva Aresta Belo, S. Devaux, P. Devynck, T. Eich, R.C. Felton, J. Flanagan, L. Frassinetti, L. Garzotti, M. Groth, S. Jachmich, A. Järvinen, E. Joffrin, M.A.H. Kempenaars, U. Kruezi, K.D. Lawson, M. Lehnen, M.J. Leyland, Y. Liu, P.J. Lomas, C.G. Lowry, S. Marsen, G.F. Matthews, G.K. McCormick, A.G. Meigs, A.W. Morris, R. Neu, I.M. Nunes, M. Oberkofler, F.G. Rimini, S. Saarelma, B. Sieglin, A.C.C. Sips, A. Sirinelli, M.F. Stamp, G.J. van Rooij, D.J. Ward, M. Wischmeier, and JET EFDA Contributors. Contrasting $\mathrm{H}$-mode behaviour with deuterium fuelling and nitrogen seeding in the all-carbon and metallic versions of JET. Nucl. Fusion, 54 (7): 073016 , 2014. URL http://stacks.iop.org/0029-5515/54/i=7/a=073016.

[6] G. Meisl, K. Schmid, O. Encke, T. Höschen, L. Gao, and Ch. Linsmeier. Implantation and erosion of nitrogen in tungsten. New J. Phys., 16 (9): 093018, 2014. URL http://stacks.iop.org/1367-2630/16/i=9/$\underline{a=093018 .}$.

[7] J. Keinonen, J. Räisänen, and A. Anttila. Diffusion of nitrogen in ion-implanted chromium and tungsten. 35 (4): 227, 1984. ISSN 0947-8396. URL http://dx.doi.org/10.1007/BF00617172.

[8] K. Schmid, A. Manhard, Ch. Linsmeier, A. Wiltner, T. Schwarz-Selinger, W. Jacob, and S. Mandl. Interaction of nitrogen plasmas with tungsten. Nucl. Fusion, 50 (2): 025006, 2010. ISSN 0029-5515.

[9] O. V. Ogorodnikova, K. Sugiyama, A. Markin, Yu. Gasparyan, V. Efimov, A. Manhard, and M. Balden. Effect of nitrogen seeding into deuterium plasma on deuterium retention in tungsten. Phys. Scr., T145 (T145): 014034, 2011. URL http://stacks.iop.org/1402-4896/2011/i=T145/a=014034.

[10] M. Oberkofler and Ch. Linsmeier. Properties of nitrogen-implanted beryllium and its interaction with energetic deuterium. Nucl. Fusion, 50 (12): 125001, 2010. URL http://stacks.iop.org/0029-5515/50/$\mathrm{i}=12 / \mathrm{a}=125001$.

[11] J. Roth, E. Tsitrone, T. Loarer, V. Philipps, S. Brezinsek, A. Loarte, G.F. Counsell, R.P. Doerner, K. Schmid, O.V. Ogorodnikova, and R.A. Causey. Tritium inventory in iter plasma-facing materials and tritium removal procedures. Plasma Phys. Control. Fusion, 50 (10): 103001, 2008. ISSN 0741-3335.

[12] M. Oberkofler, D. Alegre, F. Aumayr, S. Brezinsek, T. Dittmar, K. Dobes, D. Douai, A. Drenik, M. Könen, L.ppen, U. Kruezi, Ch. Linsmeier, C.P. Lungu, G. Meisl, M. Mozetic, C. Porosnicu, V. Rohde, and S.G. Romanelli. Plasma-wall interactions with nitrogen seeding in all-metal fusion devices: Formation of nitrides and ammonia. Fus. Eng. Des., (0): -. ISSN 0920-3796. URL http://www.sciencedirect.com/science/article/pii/S092037961500071X. 
[13] T. Dittmar, M.J. Baldwin, R.P. Doerner, D. Nishijima, M. Oberkofler, T. Schwarz-Selinger, and F. Tabares. Interaction of high flux deuterium/nitrogen plasmas with beryllium. Phys. Scr., 2011 (T145): 014009, 2011. URL http://stacks.iop.org/1402-4896/2011/i=T145/a=014009.

[14] L. Gao, W. Jacob, T. Schwarz-Selinger, and A. Manhard. Deuterium implantation into tungsten nitride: Negligible diffusion at 300 K. J. Nucl. Mater., 451: 352, August 2014. ISSN 0022-3115. URL http://www.sciencedirect.com/science/article/pii/S002231151400244X.

[15] L Gao, W. Jacob, P. Wang, U. von Toussaint, and A. Manhard. Influence of nitrogen preimplantation on deuterium retention in tungsten. Phys. Sc, 2014 (T159): 014023, 2014. URL http://stacks.iop.org/1402-4896/2014/i=T159/a=014023.

[16] J. Roth, E. Tsitrone, A. Loarte, Th. Loarer, G. Counsell, R. Neu, V. Philipps, S. Brezinsek, M. Lehnen, P. Coad, Ch. Grisolia, K. Schmid, K. Krieger, A. Kallenbach, B. Lipschultz, R. Doerner, R. Causey, V. Alimov, W. Shu, O. Ogorodnikova, A. Kirschner, G. Federici, and A. Kukushkin. Recent analysis of key plasma wall interactions issues for iter. J. Nucl. Mater., 390-391: 1-9, June 2009. ISSN 0022-3115. URL http://www.sciencedirect.com/science/article/B6TXN-4VFK84W-5/2/4d0423d14d57ae01043ccee161fe9939.

[17] T. Dittmar, M.J. Baldwin, R.P. Doerner, D. Nishijima, and T. Schwarz-Selinger. Deuterium retention in Be:N:D codeposits. J. Nucl. Mater., 438, Supplement (0): S988 - S991, 2013. ISSN 0022-3115. doi: http://dx.doi.org/10.1016/i.jnucmat.2013.01.215. URL http://www.sciencedirect.com/science/article/pii/S0022311513002237. Proceedings of the 20th International Conference on Plasma-Surface Interactions in Controlled Fusion Devices.

[18] D. Ivanova, M. Rubel, A. Widdowson, P. Petersson, J. Likonen, L. Marot, E. Alves, A. GarciaCarrasco, G. Pintsuk, and JET-EFDA Contributors. An overview of the comprehensive First Mirror Test in JET with ITER-like wall. Phys. Scr., (T159): 014011, 2014. URL http://stacks.iop.org/1402-4896/2014/$\mathrm{i}=\mathrm{T} 159 / \mathrm{a}=014011$.

[19] P. Petersson, M. Rubel, H.G. Esser, J. Likonen, S. Koivuranta, and A. Widdowson. Co-deposited layers in the divertor region of JET-ILW. J. Nucl. Mater, 463 (0): 814, August 2015. ISSN 0022-3115. URL http://www.sciencedirect.com/science/article/pii/S0022311514010204.

[20] G. De Temmerman, M.J. Baldwin, R.P. Doerner, D. Nishijima, R. Seraydarian, and K. Schmid. Insight into the co-deposition of deuterium with beryllium: Influence of the deposition conditions on the deuterium retention and release. J. Nucl. Mater., 390-391: 564-567, June 2009. ISSN 0022-3115. URL http://www.sciencedirect.com/science/article/B6TXN-4VFK84W-29/2/fb8875c89851bd25a929c2de5ddd9aa4.

[21] K. Schmid, M. Reinelt, and K. Krieger. An integrated model of impurity migration and wall composition dynamics for tokamaks. J. Nucl. Mater., 415 (1, Supplement): S284, August 2011. ISSN 00223115. URL http://www.sciencedirect.com/science/article/pii/S0022311511001528. 
[22] G. Meisl, K. Schmid, M. Oberkofler, K. Krieger, S.W. Lisgo, L. Aho-Mantila, and F. Reimold. Nitrogen retention in ASDEX Upgrade. J. N, 463 (0): 668, August 2015. ISSN 0022-3115. URL http://www.sciencedirect.com/science/article/pii/S0022311514007156.

[23] A. Herrmann, N. Jaksic, P. Leitenstern, H. Greuner, K. Krieger, P. de Marné, M. Oberkofler, V. Rohde, and G. Schall. A large divertor manipulator for ASDEX Upgrade. Fus. Eng. Des., (0): -. ISSN 0920-3796. URL http://www.sciencedirect.com/science/article/pii/S0920379615000903.

[24] K. Schmid, M. Mayer, C. Adelhelm, M. Balden, S. Lindig, and the ASDEX Upgrade team. Impact of gyro-motion and sheath acceleration on the flux distribution on rough surfaces. Nucl. Fusion, 50 (10): 105004, 2010. URL http://stacks.iop.org/0029-5515/50/i=10/a=105004.

[25] D. Neuwirth, V. Rohde, T. Schwarz-Selinger, and ASDEX Upgrade Team. Formation of ammonia during nitrogen-seeded discharges at ASDEX Upgrade. Plasma Phys. Control. Fusion, 54 (8): 085008, 2012. URL http://stacks.iop.org/0741-3335/54/i=8/a=085008.

[26] V. Rohde and M. Oberkofler. Ammonia production in nitrogen seeded plasma discharges in asdex upgrade. Journal of Nuclear Materials, 463 (0): 672-675, August 2015. ISSN 0022-3115. URL http://www.sciencedirect.com/science/article/pii/S0022311515000148.

[27] V. Rohde, D. Neuwirth, M. Oberkofler, T. Schwarz-Selinger, and the ASDEX Upgrade Team. Nitrogen balance and ammonia formation during nitrogen seeded discharges at ASDEX Upgrade. 40th EPS Conference on Plasma, 2013. URL http://ocs.ciemat.es/EPS2013PAP/pdf/P2.123.pdf.

[28] A. Drenik, M. Oberkofler, D. Alegre, U. Kruezi, S. Brezinsek, M. Mozetic, I. Nunes, M. Wischmeier, C. Giroud, G. Maddison, and C. Reux. Mass spectrometry analysis of the impurity content in N2 seeded discharges in JET-ILW. J. Nucl. Mater., 463 (0): 684, August 2015. ISSN 0022-3115. URL http://www.sciencedirect.com/science/article/pii/S0022311514010277.

[29] M. Oberkofler, D. Douai, S. Brezinsek, J.W. Coenen, T. Dittmar, A. Drenik, S.G. Romanelli, E. Joffrin, K. McCormick, M. Brix, G. Calabro, M. Clever, C. Giroud, U. Kruezi, K. Lawson, Ch. Linsmeier, A. Martin Rojo, A. Meigs, S. Marsen, R. Neu, M. Reinelt, B. Sieglin, G. Sips, M. Stamp, and F.L. Tabares. First nitrogen-seeding experiments in JET with the ITER-like Wall. J. Nucl. Mater., 438 (0): S258, July 2013. ISSN 0022-3115. URL http://www.sciencedirect.com/science/article/pii/S0022311513000494. 